\title{
Analysis of the Competitive Relationship among Commercial Banks in China from the Perspective of Game Theory
}

\author{
Jin-xia Yin ${ }^{1}$, Yun-chao Ying ${ }^{2}$, Han Jiang ${ }^{3}$ \\ ${ }^{1}$ Department of Mathematics, Jinan University, Guangzhou, China \\ ${ }^{2}$ Department of Economics, Jinan University, Guangzhou, China \\ ${ }^{3}$ Institute of China's Economic Reform \& Development, Renmin University of China, Beijing, China \\ yjx2467@foxmail.com, yingyunchao966@sina.com, danielblack@foxmail.com
}

\begin{abstract}
Commercial bank is the main body of the financial system in our country .Since China joined the WTO, an unhealthy competitive relationship has appeared among commercial banks under the effect of a variety of factors. As the game theory is an important analysis methods of economics, it can analyze these Phenomena. What's more, we can use the mathematical method to analyze and explore the results of the game theoretic model.

Index Terms - commercial bank, game theoretical model, compete, Nash equilibrium
\end{abstract}

\section{Introduction}

When a bank, as one side of the gaming, receives relatively low loan volumes, its pre-revenue will correspondingly decrease. Driven by the pursuit of short-term benefits, banks will increase the amount of loan, thus leading to the decline in the bank's income and the instability of the whole system. From the perspective of Game Theory and the introduction of Cournot Model, this paper establishes a linear dynamic model of the loan amount through the knowledge of applied mathematics algebra, and it is intended to put forward useful proposals to promote the development of commercial banks in China.

\section{Model Establishment}

\subsection{Assumptions}

(1) Human or organization is absolutely rational in the behavior of economic choice.

(2) Assuming model of duopoly discrete kinetic, and that two commercial banks were Game Party 1 and Game Party 2 respectively.

(3) Introducing the Cournot model, and establishing a linear dynamic loan volume model.

\subsection{Definition of variables}

Table1 Letters that are needed to indicate the variables required in this article

\begin{tabular}{|l|l|}
\hline$p_{i}(k)$ & The loan amount in the $\mathrm{k}_{\mathrm{th}}$ period of the $\mathrm{i}_{\mathrm{th}}$ commercial bank \\
\hline $\mathrm{P}(k)$ & The total loan amount of both commercial banks in the $\mathrm{k}_{\mathrm{th}}$ period \\
\hline$\Pi_{i}(k)$ & The margin amount in the $\mathrm{k}_{\mathrm{th}}$ period of the $\mathrm{i}_{\mathrm{th}}$ commercial bank \\
\hline$\omega_{i}(k)$ & The deposit amount of the $\mathrm{i}_{\mathrm{th}}$ commercial bank \\
\hline$\delta_{i}$ & The adjustment speed of the loan of the $\mathrm{i}_{\mathrm{th}}$ commercial bank \\
\hline$a, b$ & Positive constant, $a$ is the maximum loan rate \\
\hline$x_{i}$ & The loan risk factor of the $\mathrm{i}_{\mathrm{th}}$ commercial bank \\
\hline$y_{i}$ & Intermediate business revenue \\
\hline$c_{i}, d_{i}$ & The variable and fixed costs of the $\mathrm{i}_{\mathrm{th}}$ commercial bank \\
\hline $\boldsymbol{*}$ & The deposit rate of both commercial bank in the $\mathrm{k}_{\mathrm{th}}$ period \\
\hline $\boldsymbol{r}$ & The loan rate of both commercial bank in the $\mathrm{k}_{\mathrm{th}}$ period \\
\hline
\end{tabular}


Based on the variables above, and combined with the course of the game, we know that:

(1) The total amount of loans in the $\mathrm{k}_{\text {th }}$ period:

$$
\mathrm{P}(k)=\sum_{i=1}^{2} p_{i}(k)
$$

(2) The kth period loan interest rate is determined by total amount of loans on this period, that is:

$$
r=a-b(\mathrm{P}(k))=a-b\left(\sum_{i=1}^{2} p_{i}(k)\right)
$$

(3) Profit of a bank is relative to the bank's total amount of deposits and loans, intermediary business revenue, variable costs, fixed costs, and risk factors, then the function of the ith bank's margin $\prod_{i}$, can be expressed as:

$$
\begin{gathered}
\prod_{i}(k)=p_{i}(k)\left(r-c_{i}-x_{i}\right)-\omega_{i}(k) r^{*}+y_{i}-d_{i}, \\
(i=1,2)
\end{gathered}
$$

(4) Associated the (1) with the (3)equation, and seeking the first-order partial derivatives of equation (3) on $p_{i}$, we can have the expression of the marginal profits of the its bank as follows:

$$
\frac{\partial \prod_{i}}{\partial p_{i}}=a-b\left(2 p_{i}+p_{j}\right)-x_{i}-c_{i},(i, j=1,2 ; i \neq j)
$$

(5) We know that, when a commercial bank is relatively larger in marginal profit in a certain period, the amount of earnings brought by each unit of loan is greater. Therefore, in this case, the bank will raise the loan amount in the next period; similarly, when the profit margin is negative, the bank will lower the loan amount in the next period to reduce loss. Thus, the loan amount of each adjacent two periods should meet the following relationship:

$$
p_{i}(k+1)=p_{i}(k)+\delta_{i} p_{i} \frac{\partial \prod_{i}(k)}{\partial p_{i}(k)},(i=1,2)
$$

Associated equation (4) with (6), we obtain:

$$
\begin{gathered}
p_{i}(k+1)=p_{i}(k)+\delta_{i} p_{i}\left[a-b\left(2 p_{i}+p_{j}\right)-x_{i}-c_{i}\right], \\
(i, j=1,2 ; i \neq j)
\end{gathered}
$$

Equations (6) reflect the relationship between the loan amount in adjacent two period, and express the loan amount in $(\mathrm{k}+1)_{\text {th }}$ period as the function of the loan amount in $\mathrm{k}_{\mathrm{th}}$ period. This paper will next analyze (9) based on Dynamic Game to find the fixed point that makes the system suits the Nash equilibrium, and, combined with the knowledge of economics, draw the commercial bank competitive state corresponding to each equilibrium point.

\section{Model Analysis}

For simplicity, we assume that the system (6) satisfies the following conditions:

$$
p_{i}(k+1)=p_{i}(k) \quad(i=1,2)
$$

Associated (6) with (7), we draw that the system has 4 fixed points, namely:

The fixed points are $M_{i}(i=1,2,3,4), M_{1}=(0,0)$ means that the loan amount of both game parties is $0 ; M_{2}=\left(0, \frac{a-x_{2}-c_{2}}{2 b}\right)$ means that the loan amount of the first bank is 0 , and that of the second is $\frac{a-x_{2}-c_{2}}{2 b} ; M_{3}=\left(\frac{a-x_{1}-c_{1}}{2 b}, 0\right)$ means that the loan amount of the second bank is 0 , while the first bank' loan amount is $\frac{a-x_{1}-c_{1}}{2 b} ; M_{4}=\left(\frac{a-2\left(x_{1}+c_{1}\right)+x_{2}+c_{2}}{3 b}, \frac{a-2 x_{2}-2 c_{2}+x_{1}+c_{1}}{3 b}\right)$ mean $\mathrm{s}$ that the respective loan amount of the two banks is $\frac{a-2\left(x_{1}+c_{1}\right)+x_{2}+c_{2}}{3 b}$ and $\frac{a-2\left(x_{2}+c_{2}\right)+x_{1}+c_{1}}{3 b}$. The fixed points $M_{1}, M_{2}, M_{3}$ on the table above are all bounded equilibrium, but $M_{4}$ is Nash equilibrium point. Since loan amount of a commercial bank cannot be negative, that is, $a-x_{i}-c_{i} \geq 0,(i=1,2), a-2\left(x_{i}+c_{i}\right)+x_{j}+c_{j} \geq 0,(i, j=1,2 ; i \neq j)$

\subsection{Stability Analysis of Fixed Point $M_{1}$}

Here is the analysis of the stability of fixed points using the knowledge of Jacobi Matrix ${ }^{1}$ and its eigenvalues in the following, and the matrix in $M_{1}=(0,0)$ is:

$$
J\left(M_{1}\right)=\left(\begin{array}{cc}
1+\delta_{1}\left[a-x_{1}-c_{1}\right] & 0 \\
0 & 1+\delta_{2}\left[a-x_{2}-c_{2}\right]
\end{array}\right)
$$

Making the eigenvalues of Matrix $J\left(M_{1}\right)$ be $\lambda_{1}\left(M_{1}\right) 、 \lambda_{2}\left(M_{1}\right)$, from $\left|\lambda E-J\left(M_{1}\right)\right|=0$ (E is the identity matrix), we can draw that the two eigenvalues of the matrix are:

$\lambda_{1}\left(M_{1}\right)=1+\delta_{1}\left[a-x_{1}-c_{1}\right], \quad \lambda_{2}\left(M_{1}\right)=1+\delta_{2}\left[a-x_{2}-c_{2}\right]$. We know that $a-x_{i}-c_{i} \geq 0,(i=1,2)$, thus $\lambda_{1}\left(M_{1}\right) 、 \lambda_{2}\left(M_{1}\right) \geq 1$, therefore we can conclude that the Fixed Point $M_{1}$ is unstable. In terms of economics, the operating purpose of commercial banks is to obtain greater profits in reality, and if the loan amount of a bank is negative or just zero, then the bank might be at a loss, an outcome that no operator would like to accept. Therefore, it is impossible for the state to exist stably in commercial bank competition.

\subsection{Stability Analysis of Fixed Point $M_{2}$}

Since $M_{2}, M_{3}$ are two fixed points of the same symmetrical structure, which means the same results of stability analysis, this paper will only analyze the stability of

\footnotetext{
${ }^{1}$ Jacobi Matrix : In vector calculus, the Jacobi Matrix is a matrix arranged by first-order partial derivatives in a certain way, whose determinant is called the Jacobi Determinant. In algebraic geometry, besides, Jacobi amount of algebraic curves represents Jacobi Variety: a group of clusters along with the curve, in which the curve can be embedded. All of them are named after the mathematician Jacobi.
} 
Fixed Point $M_{2}=\left(0, \frac{a-x_{2}-c_{2}}{2 b}\right)$. The corresponding Jacobi Matrix of $M_{2}$ is:

$$
J\left(M_{2}\right)=\left(\begin{array}{cc}
1+\delta_{1}\left(\frac{a+x_{2}+c_{2}-2 x_{1}-2 c_{1}}{2}\right) & 0 \\
-b p_{2} \delta_{2} & 1+\delta_{2}\left[a-x_{2}-c_{2}\right]
\end{array}\right)
$$

Making the eigenvalues of Matrix $J\left(M_{2}\right)$ be $\lambda_{1}\left(M_{2}\right), \lambda_{2}\left(M_{2}\right)$, from $\left|\lambda E-J\left(M_{2}\right)\right|=0$ (E is the identity matrix), we can draw that the two eigenvalues of the matrix are

$\lambda_{1}\left(M_{2}\right)=1+\delta_{1}\left(\frac{a+x_{2}+c_{2}-2 x_{1}-2 c_{1}}{2}\right)>1$

$\lambda_{2}\left(M_{2}\right)=1+\delta_{2}\left[a-x_{2}-c_{2}\right]<1$

That is, the Fixed Point ${ }^{M_{2}}$ is unstable. From the angle of economics, commercial banks are in a state of free competition in financial market, and the two fixed points represent that, when one bank's loan amount is 0 , the other one's is $\frac{a-x_{1}-c_{1}}{2 b}>0$, but this case is impossible to exist, because the bank with 0 loan amount is bound to increase the volume of loan in order to increase profits, so the point actually reveals an unstable competition state between commercial banks.

\subsection{Stability Analysis of Fixed Point $M_{4}$}

Making that

$$
\begin{aligned}
M_{4} & =\left(\beta_{1}, \beta_{2}\right) \\
& =\left(\frac{a-2\left(x_{1}+c_{1}\right)+\left(x_{2}+c_{2}\right)}{3 b}, \frac{a-2\left(x_{2}+c_{2}\right)+\left(x_{1}+c_{1}\right)}{3 b}\right), \text { and since }
\end{aligned}
$$

$M_{4}$ is the Nash Equilibrium Point,

$$
J\left(M_{4}\right)=\left(\begin{array}{cc}
1-2 b \beta_{1} \delta_{1} & -b \beta_{1} \delta_{1} \\
-b \beta_{2} \delta_{2} & 1-2 b \beta_{2} \delta_{2}
\end{array}\right)
$$

Making the eigenvalues of Matrix $J\left(M_{4}\right)$ be $\lambda_{1}\left(M_{4}\right), \lambda_{2}\left(M_{4}\right)$, from $\left|\lambda E-J\left(M_{4}\right)\right|=0$ (E is the identity matrix), we can draw that the two eigenvalues of the matrix meet the formula following, ( $\operatorname{tr} J$ in the formula above is the trace of the Matrix $J$, and $\operatorname{det} J$ is the value of determinant corresponding to the matrix):

$$
\begin{aligned}
& \varphi(\lambda)=\lambda^{2}-\lambda \operatorname{tr} J+\operatorname{det} J=0 \\
& \operatorname{tr} J=2-2 b \delta_{1} \beta_{1}-2 b \delta_{2} \beta_{2} \\
& \operatorname{det} J=1+3 b^{2} \delta_{1} \delta_{2} \beta_{1} \beta_{2}-2 b \delta_{1} \beta_{1}-2 b \delta_{2} \beta_{2}
\end{aligned}
$$

Jacobi Matrix has two different eigenvalues in condition that:

$$
\Delta=(\operatorname{tr} J)^{2}-4(\operatorname{det} J)>0
$$

If the Nash Equilibrium Point meets Jury Criteria ${ }^{2}$ (necessary and sufficient conditions), then the point is stable. From Equation (11), we have the coefficients of the equation that: $a_{2}=1, a_{1}=\operatorname{tr} J, a_{0}=\operatorname{det} J$.

If Jury Principle is met, then there are three formulas as follows:

a) $\varphi(1)=1-\operatorname{tr} J+\operatorname{det} J=3 b^{2} \beta_{1} \beta_{2} \delta_{1} \delta_{2}>0$

b) $(-1)^{2} \varphi(-1)=1+\operatorname{tr} J+\operatorname{det} J$

$$
\begin{array}{r}
=4-4 b \beta_{1} \delta_{1}-4 b \beta_{2} \delta_{2} \\
+3 b^{2} \delta_{1} \delta_{2} \beta_{1} \beta_{2}>0
\end{array}
$$

c) $a_{2}>\left|a_{0}\right|$

$$
\begin{aligned}
1-|\operatorname{det} J|= & 2 b \beta_{1} \delta_{1}+2 b \beta_{2} \delta_{2} \\
& -3 b^{2} \delta_{1} \delta_{2} \beta_{1} \beta_{2}>0
\end{aligned}
$$

From the three formulas above, it is obviously that formula (14) meets Jury Criteria, and because the Nash Equilibrium Point $M_{4}$ is stable, formula (15) and (16) should be right. Thus, based on both formulas, we draw that $2 b \beta_{1} \delta_{1}+2 b \beta_{2} \delta_{2}-3 b^{2} \delta_{1} \delta_{2} \beta_{1} \beta_{2}>0>b \beta_{1} \delta_{1}+b \beta_{2} \delta_{2}-2$

The value region represented by the above formula is the stable region of the Nash Equilibrium Point. From the formula above we draw that, in this region, the stability of the system in only determined by the loan adjustment speed $\delta$ of parameter bank. Making the coordinates formed by the loan adjustment speed of both banks be $\left(\delta_{1}, \delta_{2}\right)$, we draw the formula group from the above one that:

$$
\left\{\begin{array}{c}
2 b \beta_{1} \delta_{1}+2 b \beta_{2} \delta_{2}-3 b^{2} \delta_{1} \delta_{2} \beta_{1} \beta_{2}=0 \\
b \beta_{1} \delta_{1}+b \beta_{2} \delta_{2}-2=0
\end{array}\right.
$$

From the solution of formulas above we can have the boundary value of the loan adjustment speed in a stable condition that:

$Q_{1}=\left(0, \frac{2}{2+x_{1}+c_{1}-2 x_{2}-2 c_{2}}\right), Q_{2}=\left(\frac{2}{2+x_{2}+c_{2}-2 x_{1}-2 c_{1}}, 0\right)$

These two coordinates are boundary points in the stable region of the Fixed Point ${ }^{M_{4}}$.That is, when $\left(\delta_{1}, \delta_{2}\right)$ is not in the region, the fixed point is unstable. So the stability of the Nash equilibrium points of the whole system is determined by their parameters, and there is no existence of absolute stability. In the actual economic point of view, the loan adjustment speed directly affect the amount of loan of both commercial banks, and the loan amount of two banks in the $\left(\delta_{1}, \delta_{2}\right)$ area maintains equilibrium state. In this state, both banks have a maximum

\footnotetext{
${ }^{2}$ The JURY CRITERIA: To determine the stability of discrete causal system, one should determine whether the absolute value of all the results of $\mathrm{A}(\mathrm{z})=$ 0 are less than 1 . Jury proposed list test methods, called Jury Criteria.
} 
profit. Substituting the fixed point into (3), we can have the profits of the two Banks that:

$$
\begin{aligned}
\prod_{i}(k)= & \frac{a+x_{j}+c_{j}-2 x_{i}-2 c_{i}}{3 b}\left(r-x_{i}-c_{i}\right) \\
& -\omega_{i}(k) r^{*}+y_{i}-d_{i},(i, j=1,2 ; i \neq j)
\end{aligned}
$$

However, the system did not start out with the Nash equilibrium. Before the Nash equilibrium point is reached, both parties in the game have been engaged in the dynamic game. Bank profits of each period analyze and change the loan amount of next period to get more revenue.

\section{Conclusion}

According to the analysis above, when the amount of loans is low, banks willissue more loans, thus changing the loan modify speed, resulting in the unstable situation of the system. In current condition, commercial banks can't reach to the Nash equilibrium due to their limited rationality and consequently lead to an endless dynamic game. Therefore, whether it is for commercial banks or enterprises, the situation above is not benefit for their long-term development.

\section{Acknowledgments}

Here and now, I would like to extend my sincere thanks to all those who have helped me with the paper. Meanwhile, I shall say thank you to the ICEMSS 2013 conference which has provided such a wonderful platform for us to show ourselves. Thank you very much.

\section{Reference}

[1] Brissimis S N, Delis M D. Bank-level estimates of market power. European Journal of Operational Research, 2011, 212(3): 508-517.

[2] Skully M, Perera S. Bank market power and revenue diversification: Evidence from selected ASEAN countries. Journal of Asian Economics, 2012.

[3] Chen W. WTO: Time's Up for Chinese Banks-China's Banking Reform and Non-Performing Loan Disposal. Chi. J. Int'l L., 2006, 7: 239. 